Número 45 (diciembre de 2021) | No. 45 (December 2021)

$$
\text { Artículos | Articles }
$$

Apellidos, nombre y filiación institucional

Surname, name and institutional affiliation

Gonzalo Ana Dobratinich

CONICET. Facultad de Derecho.

Universidad de Buenos Aires

María del Carmen Barraco Avilés

Instituto de Derechos Humanos "Gregorio

Peces-Barba".

Departamento de Derecho Internacional Público, Eclesiástico y Filosofía del Derecho.

Universidad Carlos III de Madrid psicosocial como situaciones de vulnerabilidad
Título

Title

\section{Correo electrónico}

Páginas

Email

Pages

Relaciones entre derecho y literatura en la historia argentina. Propuestas para su estudio desde la filosofía del derecho.

Relations between law and literature in argentine history. Proposals for your study from the philosophy of law.

La discapacidad intelectual y la discapacidad

Intellectual and Psychosocial Disability as Situations of Vulnerability

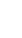


Diego Borbón

Universidad Externado de Colombia

\section{Federico Fernández Crehuet}

Departamento de Filosofía del derecho

Universidad de Granada

Nacho Hernández Moreno

Facultad de Turismo y Relaciones

Internacionales, Universidad de Murcia

Universidad de Murcia
Incompatibilismo humanista: Una contrapropuesta del neuroabolicionismo penal

Humanistic incompatibilism: A counterproposal of ternado.edu.co diego.borbon01@est.uex penal neuroabolitionism

La Introducción a la ciencia del derecho de Radbruch como síntoma de una época

Radbruch's Introduction to the Science of Law as the crehuet@ugr.es Spirit of a Time

La atención humanitaria como alternativa viable y eficaz a la detención migratoria: por una política migratoria de retorno centrada en la integración

Humanitarian assistance as a viable and effective alternative to immigration detention: for an integration-centered immigration policy on returns ijhm86@gmail.com

$100-126$ 
Oscar Pérez de la Fuente

Universidad Carlos III de Madrid

Aurelio de Prada García

Universidad Rey Juan Carlos

Elena Prats

Universidad de Uppsala (Suecia)
Libertad de expresión y performatividad. Análisis de algunos casos judiciales sobre lenguaje del odio y/o simbólico

Free speech and performativity. Analysis on some oscar@der-pu.uc3m.es

$127-156$ judicial case on hate speech and/or symbolic speech

Los prejuicios no percibidos y el caso Sócrates: un ejemplo paradigmático.

The hidden prejudices and the Socrates case: a aurelio.deprada@urjc.es paradigmatic example.

Las formas de lo arbitrario: Los altos y bajos de Towards a Theory of Arbitrary Law-making in Migration Policy de Patricia Mindus

The Forms of Arbitrariness: The Ins and Outs of Towards a Theory of Arbitrary Law-making in Migration Policy by Patricia Mindus 
Análisis sociojurídico de las transformaciones en la provisión de seguridad. Austeridad y aporofobia como racionalidades estructurantes de la

David Vila-Viñas

Departamento de Filosofía del Derecho Universidad de Málaga gobernabilidad contemporánea en España

Socio-legal analysis of the transformations in the provision of security. Austerity and aporophobia as structuring rationalities of the contemporary governmentality in Spain. dvila@unizar.es

$195-226$ 
Sección especial sobre bienes comunes | Special section on Common Goods

Apellidos, nombre y filiación institucional

Surname, name and institutional affiliation

Luis Lloredo Alix y Ricardo Cueva

Universidad Autónoma de Madrid

Ermanno Vitale

Dipartimento di Scienze Economiche

Politiche

Università della Valle d'Aosta (Italia)

\section{Luis Lloredo Alix}

Departamento de derecho público y filosofía jurídica

Universidad Autónoma de Madrid

\section{Título}

Title

Presentración de la sección especial sobre bienes comunes

Foreword to the special section on Common Goods

luislloredo@gmail.com

ricardo.cueva@uam.es

Más allá de la retórica. ¿Qué queda en pie del bonum commune?

Beyond rethorics. Is there anything left from the e.vitale@univda.it concept of bonum commune?

Los bienes comunes como aproximación materialista al problema del bien común: una réplica a Ermanno Vitale

The commons as a materialist approach to the luislloredo@gmail.com problem of common good: An answer to Ermanno Vitale
$227-231$

Páginas

Pages

$250-283$ 
Daniel J. García López

Departamento de Filosofía del Derecho

Universidad de Granada

Marco Aparicio Wilhelmi

Facultad de Derecho

Universitat de Girona

Eduardo Melero Alonso

Área de Derecho Administrativo

Universidad Autónoma de Madrid
Los bienes comunes en el pensamiento de Stefano

Rodotà

The commons goods in the thought of Stefano danieljgl@ugr.es

Rodotà

Más allá de lo constituido. Lo común como hipótesis jurídico-constitucional

Beyond the constituted. The commons as a legal- marco.aparicio@udg.edu 302-332 constitutional hypothesis

El derecho de la globalización neoliberal como marco limitador de la promoción de los comunes. Un análisis desde el derecho público

The law of neoliberal globalisation as a limiting eduardo.melero@uam.es

333-356 framework for the promotion of the commons. A public law analysis 
Apellidos, nombre y filiación institucional Surname, name and institutional affiliation

Francisco Javier Ansuátegui Roig

Universidad Carlos III de Madrid

Vicente Bellver Capella

Departamento de Filosofía del Derecho y Política

Universitat de València

\section{Martí Colom Nicolau}

Universitat de Barcelona

Clara Esteve Jordà

Departamento de Derecho Público

Universitat Rovira i Virgili

\section{Recensiones | Book Reviews \\ Título \\ Title}

Tincani, Persio. Identità e meraviglia. Cinque scritti brevi di diritto, politica e letteratura. Milano: l'Ornitorinco edizioni, 2020

javofil@der-pu.uc3m.es

Ballesteros, Jesús. Domeñar las finanzas, cuidar la naturaleza. Valencia: Tirant lo Blanch, 2021.

Lara Amat y León, Joan (ed.). La ciudadanía y lo político. Ciudadanía y crisis de la democracia liberal en un mundo en transformación. Lima: ONPE, 2020.

Crétois, Pierre. La part commune. Critique de la propriété privée. Paris: Éditions Amsterdam, 2020.
$357-366$

Vicente.Bellver@uv.es

\section{Correo electrónico \\ Páginas \\ Pages \\ Email}

marti.clm@gmail.com

$376-382$

clara.esteve@urv.cat

$383-386$ 
Jesús Mora

Universidad Carlos III de Madrid
Pérez de la Fuente, Oscar; Falcão, Monique y Oliva Martínez, J. Daniel (eds.) Struggles for Recognition. Cultural Pluralism and Rights of Minorities. Madrid: Dykinson, 2020.

jesuspmora14@gmail.co $\mathrm{m}$

$387-392$ alfonsorufino.ortega@uv a.es Belloso Martín, N. (Dir.). Sociedad plural y nuevos retos del Derecho. Cizur Menor (Navarra): Thomson Reuters Aranzadi, 2021.

García Manrique, Ricardo. Se vende cuerpo. El debate sobre la venta de órganos. Barcelona: Herder, 2021.

lukas.romero@uv.es

$401-406$

Belloni, Ilario y Magneschi, Chiara. Derecho salvaje. Hombres y animales entre estado de naturaleza y michele.zezza@for.unipi. civilización jurídica. Madrid: Reus, 2020.
Michele Zezza

Universidade de São Paulo (USP), Brasil 


\section{Estadísticas del número 45 de Cuadernos Electrónicos de Filosofía del Derecho}

Statistics of No. 45 of Cuadernos Electrónicos de Filosofía del Derecho

Artículos recibidos | Articles received: 24

Publicados | Published: 15 (62,5\%)

Rechazados | Rejected: 9 (37,5\%)

Plazo medio de espera hasta la comunicación de la decisión editorial| Average waiting time on editor's decision: 6 meses | months 